\title{
An improvement of a lemma from Gauss's first proof of quadratic reciprocity \\ by
}

\section{A. SCHINZEL and M. SKAŁBA}

Summary. An upper estimate is given for the least prime $q$ such that $(d / q)=1$ and $(p / q)=-1$, where $d \neq 0$ is a given integer and $p$ is a given prime satisfying $p \equiv 1(\bmod 8)$ and $(d / p)=1$.

In his proof (and the first proof altogether) of the quadratic reciprocity law Gauss applied the following statement:

(*) For every prime $p$ of the form $8 k+1$ there exists a prime $q<p$ satisfying

$$
\left(\frac{p}{q}\right)=-1 .
$$

For $p \equiv 1(\bmod 4)$ and $q>2, \sqrt{1}$ is equivalent to

$$
\left(\frac{q}{p}\right)=-1,
$$

and it is a well known problem to estimate the least prime $q$ for which (2) holds. The best unconditional result is due to Burgess [1]:

$$
q<p^{\frac{1}{4 \sqrt{e}}+\varepsilon}
$$

for every $\varepsilon>0$ and $p>p_{0}(\varepsilon)$.

We shall improve, at least asymptotically, the statement $(*)$ by proving

THEOREM 1. For every integer $d \neq 0$, every real number $\varepsilon>0$ and every prime $p>p_{0}(d, \varepsilon)$ with $p \equiv 1(\bmod 8)$ and $(d / p)=1$ there exists a prime

2010 Mathematics Subject Classification: Primary 11A15, 11L40.

Key words and phrases: quadratic residue, quadratic character.

Received 23 March 2017.

Published online 18 April 2017. 
$q<p^{2 / 3+\varepsilon}$ such that

$$
\left(\frac{d}{q}\right)=1 \quad \text { and } \quad\left(\frac{p}{q}\right)=-1 .
$$

For $d=-1,2$ and $\varepsilon=1 / 3$ one can take $p_{0}(d, \varepsilon)=0$.

The proof of the Theorem is based on three lemmas.

Lemma 1. Assume that $p>2$,

$$
\left(\frac{d}{p}\right)=1
$$

and $M>0$. The number of pairs $(x, y) \in \mathbb{Z}^{2} \cap(0, M]^{2}$ such that

$$
x^{2}-d y^{2} \equiv 0(\bmod p)
$$

is $2 M^{2} / p+O(M)$, where the constant in the O-symbol is absolute.

Proof. Since $d \equiv \delta^{2}$ and $\delta \not \equiv-\delta(\bmod p),(5)$ is equivalent to

$$
x \equiv \pm \delta y(\bmod p) .
$$

For every $y \in \mathbb{Z} \cap(0, M]$ there are $2 M / p+O(1)$ integers $x \in(0, M]$ satisfying (6). Hence the lemma.

Let $\nu_{q}(d)$ be the exact exponent with which the prime $q$ divides $d$.

Lemma 2. Let $d \neq 0$ be a cube free integer and let $q_{1}, \ldots, q_{k}$ be all distinct prime divisors of $d$ such that

$$
\left(\frac{q_{i}}{p}\right)=-1
$$

Let $e_{i}=\nu_{q_{i}}(d)(1 \leq i \leq k)$ and $S$ be a subset of $\{1, \ldots, k\}$. The number $N(S)$ of pairs $(x, y) \in \mathbb{Z}^{2} \cap(0, M]^{2}$ such that

$$
x^{2}-d y^{2} \not \equiv 0(\bmod p), \quad \nu_{q_{i}}\left(x^{2}-d y^{2}\right) \equiv 1(\bmod 2) \quad(i \in S)
$$

equals

$$
M^{2}\left(1-\frac{2}{p}\right) \prod_{i \in S} x_{i}+O(M)
$$

for some $x_{i}<1 / 2$, where the constant in the O-symbol depends on $d$.

Proof. All $q_{i}$ are odd, because of the condition $p \equiv 1(\bmod 8)$. The condition $(7)$ is equivalent to the conjunction of $x^{2}-d y^{2} \not \equiv 0(\bmod p)$ and

$$
\nu_{q_{i}}(x)>\nu_{q_{i}}(y) \quad \text { if } e_{i}=1,
$$

$\nu_{q_{i}}(x)=1+\nu_{q_{i}}(y), \nu_{q_{i}}\left(x^{2} q_{i}^{-2 \nu_{q_{i}}(x)}-d q_{i}^{-2} y^{2} q_{i}^{-2 \nu_{q_{i}}(y)}\right) \equiv 1(\bmod 2)$ if $e_{i}=2$.

In order to get the estimate we set 


$$
\begin{array}{ll}
x_{i}=\frac{1}{q_{i}+1} & \text { if } e_{i}=1, \\
x_{i}=\frac{2}{\left(q_{i}+1\right)^{2}} & \text { if } e_{i}=2,\left(\frac{d q_{i}^{-2}}{q_{i}}\right)=1, \\
x_{i}=0 & \text { if } e_{i}=2,\left(\frac{d q_{i}^{-2}}{q_{i}}\right)=-1,
\end{array}
$$

and apply the Chinese Remainder Theorem together with Lemma 1. We only give the rationale for the formula on $x_{i}$ in case $e_{i}=2$, because it is more involved.

Let $x=q_{i}^{\nu_{q_{i}}(x)} \alpha, y=q_{i}^{\nu_{q_{i}}(y)} \beta$ and $q_{i} \nmid \alpha \beta$. Then the second of conditions (7) is equivalent to

$$
\nu_{q_{i}}(x)=1+\nu_{q_{i}}(y) \quad \text { and } \quad \nu_{q_{i}}\left(\alpha^{2}-d^{\prime} \beta^{2}\right) \equiv 1(\bmod 2) \quad \text { where } d^{\prime}=d / q_{i}^{2} .
$$

Let $B=\mathbb{Z}^{2} \cap(0, M]^{2}, B^{\prime}=\left\{(\alpha, \beta) \in B \mid q_{i} \nmid \alpha \beta\right\}$. First if $\left(d^{\prime} / q_{i}\right)=-1$, then

$$
\left\{(x, y) \in B \mid \nu_{q_{i}}\left(x^{2}-d y^{2}\right) \equiv 1(\bmod 2)\right\}=\emptyset,
$$

so we set $x_{i}=0$. Secondly, if $\left(d^{\prime} / q_{i}\right)=1$, then

and for any fixed $k \geq 1$,

$$
\operatorname{card} B^{\prime}=\left(\frac{q_{i}-1}{q_{i}}\right)^{2} \operatorname{card} B+O(M),
$$

$$
\begin{aligned}
b_{k} & :=\operatorname{card}\left\{(\alpha, \beta) \in B^{\prime} \mid \nu_{q_{i}}\left(\alpha^{2}-d^{\prime} \beta^{2}\right) \geq k\right\} \\
& =\operatorname{card} B^{\prime} \cdot \frac{2\left(q_{i}^{k}-q_{i}^{k-1}\right)}{\left(q_{i}^{k}-q_{i}^{k-1}\right)^{2}}+O(M) .
\end{aligned}
$$

Hence

$$
\begin{aligned}
& \operatorname{card}\left\{(\alpha, \beta) \in B^{\prime} \mid \nu_{q_{i}}\left(\alpha^{2}-d^{\prime} \beta^{2}\right) \equiv 1(\bmod 2)\right\}=b_{1}-b_{2}+b_{3}-\cdots \\
& \quad=\frac{2}{q_{i}-1}\left(1-\frac{1}{q_{i}}+\frac{1}{q_{i}^{2}}-\cdots\right) \operatorname{card} B^{\prime}+O(M)=\frac{2 q_{i}}{q_{i}^{2}-1} \operatorname{card} B^{\prime}+O(M),
\end{aligned}
$$

and finally

$$
\begin{aligned}
\operatorname{card}\{(x, y) \in B \mid & \left.\nu_{q_{i}}\left(x^{2}-d y^{2}\right) \equiv 1(\bmod 2)\right\} \\
& =\left(\frac{1}{q_{i}}+\frac{1}{q_{i}^{3}}+\cdots\right) \frac{2 q_{i}}{q_{i}^{2}-1}\left(\frac{q_{i}-1}{q_{i}}\right)^{2} \operatorname{cardB}+O(M) \\
& =\frac{2}{(q+1)^{2}} \operatorname{card} B+O(M),
\end{aligned}
$$

which justifies the definition of $x_{i}$ in case $\left(d^{\prime} / q_{i}\right)=1$.

Lemma 3. Let $f(x, y)=x^{2}+a x y+b y^{2}$ be an integral quadratic form reducible $\bmod p$, but not congruent $\bmod p$ to a perfect square. Let $\chi$ be a non-principal character mod $p$ and $B=\mathbb{Z}^{2} \cap(0, M]^{2}$. For every $\varepsilon>0$ there 
exists $\delta>0$ such that

$$
\sum_{(x, y) \in B} \chi(f(x, y))=O\left(M^{2} p^{-\delta}\right) \quad \text { for every } M>p^{1 / 3+\varepsilon} .
$$

Proof. See Burgess [2].

Proof of the Theorem. Without loss of generality we can assume that for each prime $q$ dividing $d$ one has $\nu_{q}(d) \leq 2$. In Lemma 3 , set $f(x, y)=x^{2}-d y^{2}$, $\chi=(\cdot / p), M=p^{(1+\varepsilon) / 3}$. By the condition $(d / p)=1$ the assumptions of the lemma are satisfied. By the lemma, (8) holds for a certain $\delta>0$. On the other hand, by Lemma 1 $\chi\left(x^{2}-d y^{2}\right)=0$ for at most $2 M^{2} / p+O(M)$ terms of the sum over $B$. Let $q_{1}, \ldots, q_{k}$ be all distinct prime divisors of $d$ such that $\left(q_{i} / p\right)=-1$, and for $S \subset\{1, \ldots, k\}$ let $N(S)$ be as in Lemma 2. Assuming that there is no prime $q<p^{2 / 3+\varepsilon}$ satisfying $(3)$, the number of terms in the sum $(8)$ in which $\chi\left(x^{2}-d y^{2}\right)=-1$ is

$$
\begin{aligned}
M^{2}\left(1-\frac{2}{p}\right) \sum_{\text {card } S \text { odd }} & \prod_{i \in S} x_{i} \prod_{i \notin S}\left(1-x_{i}\right)+O(M) \\
& =M^{2}\left(1-\frac{2}{p}\right)\left(\frac{1}{2}-\frac{1}{2} \prod_{i=1}^{k}\left(1-2 x_{i}\right)\right)+O(M),
\end{aligned}
$$

which can be justified as follows: for given $l$ natural numbers $i_{1}<\cdots<i_{l} \leq k$ we compare the coefficients of $x_{i_{1}} \cdots x_{i_{l}}$; on the left-hand side the relevant coefficient equals

$$
M^{2}\left(1-\frac{2}{p}\right)(-1)^{l-1} \sum_{j \equiv 1(\bmod 2)}\left(\begin{array}{l}
l \\
j
\end{array}\right)=M^{2}\left(1-\frac{2}{p}\right)(-2)^{l-1}
$$

and on the right-hand side the coefficient has the same value. Now we obtain

$$
\begin{aligned}
\sum_{(x, y) \in B} \chi(f(x, y))= & \operatorname{card} B-M^{2} \frac{2}{p}-O(M) \\
& -M^{2}\left(1-\frac{2}{p}\right)\left(1-\prod_{i=1}^{k}\left(1-2 x_{i}\right)\right)+O(M) \\
= & M^{2}\left(1-\frac{2}{p}\right) \prod_{i=1}^{k}\left(1-2 x_{i}\right)+O(M),
\end{aligned}
$$

which is not $O\left(M^{2} p^{-\delta}\right)$. The contradiction proves the existence of a prime $q$ satisfying (3).

\section{References}

[1] D. A. Burgess, The distribution of quadratic residues and non-residues, Mathematika 4 (1957), 106-112. 
[2] D. A. Burgess, A note on character sums of binary quadratic forms, J. London Math. Soc. 43 (1968), 271-274.

A. Schinzel

M. Skałba

Institute of Mathematics

Institute of Mathematics

Polish Academy of Sciences

University of Warsaw

Śniadeckich 8

Banacha 2

00-656 Warszawa, Poland

02-097 Warszawa, Poland

E-mail: schinzel@impan.pl

E-mail: skalba@mimuw.edu.pl 
